\title{
Incidencia de Tecnología RFID en Gestión Bibliotecaria en una IES de Guayaquil
}

\section{Library Management Incidence of RFID Technology in a Guayaquil's Upper Education Institution}

Vilma Alvarez-Intriago ${ }^{a}$, Israel Vega Arguello ${ }^{b}$, Joe Echeverria-Reyes $^{c}$

\author{
INFORMACIÓN DEL \\ ARTÍCULO \\ Fecha de recepción: 08 de febrero del \\ 2017 \\ Fecha de aceptación: 10 de marzo del \\ 2017
}

\begin{abstract}
Resumen
El gran desafío de las bibliotecas es poder llevar un correcto control sobre sus ejemplares por medio de inventarios eficientes. Por ello, se han creado soluciones para identificarlos de forma rápida. Una de ellas, es la identificación por radiofrecuencia (RFID) cuya técnica se basa en la captura de datos de forma inalámbrica, permitiendo realizar lecturas más rápidas y precisas. El presente trabajo, se enfoca en integrar información obtenida por RFID de libros vigentes al sistema de administración bibliotecario de una Institución de Educación Superior (IES), a través de una aplicación web de código abierto, con el objetivo de que los operadores puedan realizar el proceso de inventario del material bibliográfico de forma eficiente. Para ello, se realiza el levantamiento de requerimientos, luego el desarrollo del sistema integrador y finalmente pruebas de funcionamiento. Como resultado se evidenció que utilizando tecnología RFID la gestión de inventario de la biblioteca mejora aproximadamente el $70 \%$.
\end{abstract}

Palabras Clave

Tecnología RFID, Biblioteca, inventario, modelo MVC

Clasificación JEL: O32, O33, M15. $b$ Ingeniero en Sistemas, Universidad Espíritu Santo - Ecuador

E-mail: israelvegaarguello@gmail.com

\footnotetext{
Magister en Administración de Empresas, ESPAE. Ingeniero en Computación, ESPOL. Docente de la Facultad de Postgrado, Universidad Espíritu Santo - Ecuador

E-mail: jecheverria@uees.edu.ec
}

\begin{abstract}
Correct specimen control through inventory efficiency is the great challenge of all libraries. Quick identification solutions have been created for this purpose. Radio frequency identification (RFID) is one. It is a technique based on wireless data capture, which allows faster and more accurate readings. The present work focuses on integrating current book information obtained by using RFID in the library administration system of a higher education institution (IES), through an open source web application; this purports to allow operators to efficiently perform a bibliographic material inventory process. To do this, requirements are raised; the integrator system is then developed and functionally tested. A 70\% improvement of library inventory management was evidenced as a result of using RFID technology.
\end{abstract}

Keywords: RFID technology, Library, inventory, model MVC

JEL Classification: O32, O33, M15. 


\section{Introducción}

En la actualidad, la ejecución de tareas de forma manual y procedimientos repetitivos se tornan incómodos e ineficientes. Es por ello, que los procesos automatizados toman un rol cada vez más protagónico dentro de una organización. Debido a esto, las organizaciones han incurrido en extensas búsquedas de soluciones que les ayude a resolver dichos problemas. De acuerdo a Davara (2010), uno de los problemas claves que se presenta en una organización de cualquier índole es la administración de los inventarios, cuando el identificador del objeto no coincide con la información del reporte se genera una tarea adicional de búsqueda y averiguación; lo cual provoca no solo malestar sino también retrasos en la productividad del personal.

El problema se agudiza cuando se trata de una organización cuyo giro de negocio se basa en la administración de grandes volúmenes de productos o artículos. Es aquí, donde se encasillan las empresas manufactureras y las bibliotecas, cuya función principal es la gestión de artículos. Por lo que, la automatización del proceso de identificación de ejemplares se convierte en un requisito esencial para poder efectuar la administración de forma correcta y el mantenimiento de la información.

De acuerdo a Marimón (2010), es necesario recalcar que, la preocupación de las bibliotecas durante los últimos años ha girado en torno a la búsqueda de soluciones tecnológicas que les permitan realizar sus procesos de una forma eficaz y eficiente. Ya que, debido al volumen de libros, la alta rotación y la incorrecta manipulación de los ejemplares se dificulta y se atrasa los tiempos de administración del material bibliográfico. Por otro lado, existen diversas tecnologías que se enfocan principalmente en la identificación de productos, entre ellas la más popular es la de código de barras. Sin embargo, León (2011) sostiene que a pesar que el código de barras ha sido el principal medio de identificación de productos durante los últimos 25 años, es probable que quede sustituido por la tecnología RFID, gracias a los múltiples beneficios que ofrece a las organizaciones.

Es importante recalcar que de acuerdo a Muñuzuri, Escudero, Rodríguez \& Rubia (2012) los sistemas RFID son implementados en empresas de todo el mundo creando soluciones eficientes y sostenibles y logra disminuir los errores humanos, el tiempo excesivo de conteo de inventarios y disminuye las pérdidas de los activos. Sin embargo, las empresas de logística y cadenas de suministro son considerados como los principales ejemplos de aplicaciones RFID, ya que la mayoría de los proyectos piloto se da en los países desarrollados o industrializados Adhiarna, Hwang y Rho, (2011).

Según Correa, Álvarez \& Gómez (2010), en el mercado, principalmente en el ámbito manufacturero existen una gran variedad de soluciones para la identificación de productos, entre ellas se tiene el código de barras, los códigos QR, entre otros. Todos ellos con la finalidad de poder capturar los datos del producto $\mathrm{y}$

INVESTIGATIO No. 9, marzo 2017, pp. 51-66

(C) Universidad Espíritu Santo - UEES

ISSN: 1390 - 6399 
transferirlos a un sistema de gestión para su procesamiento. Sin embargo, sistema de Gestion (2015), afirma que la tecnología RFID a diferencia de sus competidores, permite la obtención de inventarios rápidos $\mathrm{y}$ fáciles, estadísticas eficaces $\mathrm{y}$ etiquetas personalizables, lo que le genera un valor agregado a las empresas que se han visto beneficiadas por su utilización en sus cadenas de procesos. Tal es el caso de las empresas colombianas que gracias a la implementación de RFID en sus fábricas en los últimos años se han potenciado principalmente por el almacenamiento y distribución de grandes volúmenes de mercadería hacia todas las partes de América del Sur (Llamazares, 2013). De igual manera, según Villareal, Arango \& Moreno (2012) sostienen que las empresas que gestionan extensos volúmenes de artículos son las que mayormente se han visto beneficiadas, puesto que con RFID la entrega de los datos de un producto a lo largo de una cadena de abastecimiento ya no se torna difícil. Así mismo, Jiménez (2013) agrega que hoy en día se considera a la tecnología RFID como una de las principales herramientas tecnológicas existentes para acelerar la captura de datos de un proceso en donde se involucran extensas cantidades de productos y se refiere a RFID como la solución rentable, innovadora $y$ confiable, que promete beneficiar a las empresas cuyo modelo de negocio se enfoque en la identificación y trazabilidad de artículos.

Un notable ejemplo, Mejía, Yepes \& Arango (2013) lo enuncian en la empresa Crystal Vestimundo, que realizó una prueba de implementación de la tecnolo- gía RFID en su centro de distribución en la ciudad de Medellín; la prueba consistía en realizar la trazabilidad del envío de su mercadería desde la matriz hacia sus diferentes sucursales, obteniendo como resultado la reducción de 24 minutos del registro de una caja de 150 prendas, a tan solo 4 minutos. Un caso similar, es el de Almacenes Éxitos que realizó tres pruebas pilotos, intentando integrar grandes proveedores de esta tecnología y obteniendo como resultado el mejoramiento de la eficiencia operativa de la distribución de los productos, hacia las diferentes sucursales (Swedberg, 2016).

Así también, Texas Instrument (2014) afirma que Lufthansa Technik, la empresa brasileña ha incursionado en el campo de la identificación por radiofrecuencia y logró una reducción en un $80 \%$ en la gestión de sus inventarios. Texas Instrument (2015) ha emitido un comunicado, donde informa que el medio automotriz también se ha visto favorecido por la tecnología RFID, ya que la empresa Texas Instruments ha desarrollado aplicaciones para controlar el acceso de vehículos para parqueaderos, lo que permite la optimización del tiempo y mejor uso del espacio.

De igual forma, Sierra (2012) brinda el ejemplo de la instalación de RFID en la biblioteca de ESADE, Escuela Superior de Administración y Dirección de Empresas en Sant Cugat del Vallés, que implementó la tecnología en el 2009 con el objetivo de incrementar la eficiencia de los procesos, para mejorar los servicios a los usuarios y asegurar el control del material bibliográfico. 
De acuerdo a Cruz (2012), se calcula que el $5 \%$ de las bibliotecas con más de 100.000 ejemplares utilizan RFID en alguno de sus procesos, siendo Europa el continente con la mayor cantidad de instalaciones abarcando desde bibliotecas pequeñas hasta las patrimoniales de gran nivel, como lo es la del Vaticano que cuenta con 1,5 millones de ejemplares aproximadamente. Así también es el caso de la biblioteca pública del distrito de Villa de Vallecas de Madrid que con la implementación de RFID ha podido administrar de una forma eficiente cerca de 50.000 libros y 15.000 unidades multimedia.

Lo anteriormente expuesto recalca que las bibliotecas se han visto beneficiadas por la tecnología RFID, reduciendo problemas relacionados a generación de inventarios provocados por errores humanos y disminuyendo los tiempos de ejecución. Así, el presente artículo tiene como objetivo realizar un proceso de inventario eficiente de los ejemplares de la biblioteca de la IES a través de la tecnología RFID y el desarrollo de una aplicación web.

\section{Marco Teórico}

\section{Tecnología RFID.}

De acuerdo a León (2011), la tecnología RFID es una transferencia y recuperación de datos sin ningún tipo de contacto físico, cuyo objetivo principal es transmitir la identidad de un objeto. Su funcionalidad se representa en la Figura 1.

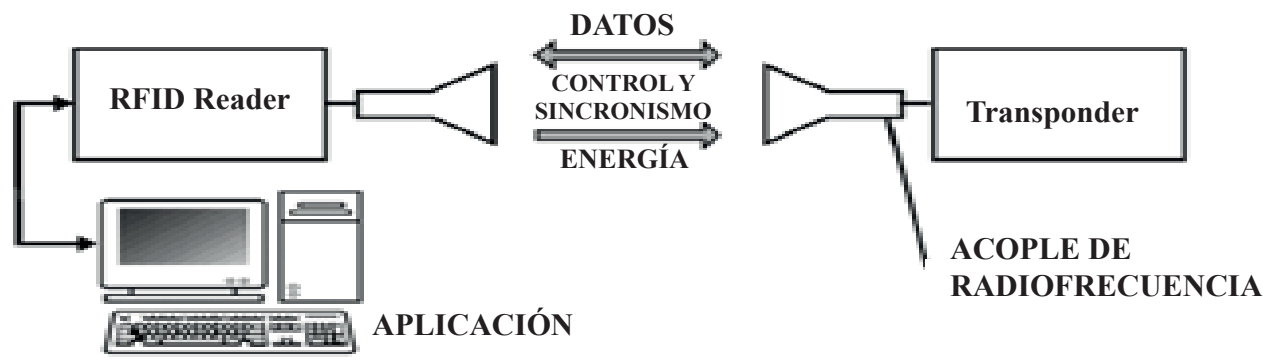

Figura 1. Funcionamiento de tecnología RFID. León (2011).

De igual manera, Sistemas Informaticos (2012), sostiene que es un proceso de captura de datos de forma automática sin línea de visión y sin contacto alguno, es decir que trabaja a través de ondas, con la finalidad de obtener la información serializada de cualquier tipo de artículo. Por lo que, busca dejar a un lado las deficiencias que el código de barras no pudo resolver, permitiendo obtener informa- ción en tiempo real no solo identificando el producto, sino que también guarda información sobre comportamientos sobre los cuales se podrá después inferir escenarios susceptibles de modelación y optimización. Así mismo, Fontalvo, Mendoza \& Visbal (2014) definen a RFID como una tecnología que permite la identificación de productos, alimentos, objetos e inclusive animales por medio de 
la captura de datos de forma inalámbrica, lo que produce que el proceso sea rápido $\mathrm{e}$ inequívoco, obteniendo el mayor provecho de la movilidad entre los equipos y el desplazamiento de los operadores. De la misma manera, Ramírez \& Meléndez (2014) sostienen que el uso de RFID aumenta la eficiencia y velocidad de los procesos proporcionando información exacta y oportuna. Así que, se ha convertido en una de las principales herramientas en la cadena de suministros.

Según García, Barbeau \& Kranakis (2010), la tecnología RFID poseen una estructura basada en el Código Electrónico de Producto (EPC) la cual mantiene una arquitectura distribuida de bajo costo, debido a los reducidos precios de sus etiquetas (Meliá, 2011). Es por esto, que teniendo en cuenta que la funcionalidad de RFID tiene como objetivo principal la automatización de procesos, a continuación se definirán conceptos relacionados.

\section{Gestión de procesos.}

Alonso (2010) sostiene que en el ámbito organizacional cualquier tarea $\mathrm{o}$ actividad puede ser encuadrada en un algún proceso, incluso aunque éste no haya sido definido formalmente, por lo que las estadísticas indican que cada vez son mayores las empresas que adoptan sistemas de gestión basados en procesos.

Por otro lado, Mallar (2010) hace énfasis en definir a un proceso como un conjunto de tareas interrelacionadas que requieren de entradas para que por medio de actividades específicas se las puedan transformar y agregarles valor a los elementos salientes. Así, en una gestión de inventario, los procesos comienzan con las entradas que son los datos de los libros y como salida se obtendrá información útil para la administración de los ejemplares (Jechlitschek, 2006).

\section{Lenguaje de programación.}

Acorde con ese criterio Villarejo (2010) sostiene que, para poder llevar a cabo el desarrollo de una solución tecnológica es necesario el lenguaje de programación correcto, porque es el que permitirá el vínculo entre el ordenador y el usuario. Uno de los lenguajes que permite el desarrollo de aplicaciones web, es PHP, el cual ha tenido mayor expansión en los últimos diez años por su robustez y su ejecución sin licencia propietaria.

De igual manera, Jabba, Alcocer \& Rojas (2011) se refieren a PHP como un lenguaje de código abierto, fácil de aprender y con un fuerte enfoque a la programación web, lo que facilita la conexión con las bodegas de datos. Equipo Minero (2014) afirma que es posible que las organizaciones transciendan en su campo y tengan un mejor desempeño en sus actividades si los niveles de satisfacción de los usuarios aumentan de acuerdo a la rapidez de las transacciones. Por esto, Guamán \& Recalvode (2013) sugiere que la programación se la realice basándose en el modelo vista controlador (MVC) cuya principal característica es el trabajo en capas para dividir el código en base a su función. De la misma manera, de acuerdo a Martínez (2010), la programación basándose en $\mathrm{MVC}$, permite que sea 
más eficiente. Así también Camarena, Trueba, Martínez \& López (2012), afirma que este modelo se basa en separar en tres capas a todo el proyecto: lógica de control, lógica de negocio y lógica de presentación. Debido a que el proyecto recibirá datos providentes de varias fuentes, es preciso definir el término de bodega de datos.

\section{Bodega de datos y base de datos.}

Calabria (2011) denomina bodegas de datos a la integración de los datos consolidados y que se encuentran acopiados en un dispositivo de memoria no volátil y que provienen de distintas fuentes. Por lo tanto, se diferencian de las bases de datos, porque no procesan ningún tipo de dato, sino que sirven como repositorios temporales.

Por otro lado, Rubinos \& Nuevo (2011) definen a una base de datos como una serie de datos organizados y relacionados entre sí, los cuales son recolectados y explotados por los sistemas de información de una empresa o un negocio en particular y se componen de una o más tablas y cada tabla se forma de filas y columnas. Es decir, que tienen una estructura lógica definida, la cual se denomina diseño de la base de datos, que permite la organización de los datos para una mejor organización y comprensión (Zapata, González \& Chaverra, 2011).

De acuerdo a Wei \& Rui (2013), el diseño de la base de datos se refiere a la agrupación de la tabla de los datos estadísticos y la tabla de datos clasificados acorde a los módulos de las funcionalida- des del sistema. Por lo que, en la actualidad existen diferentes motores de bases de datos, todos ellos varían de acuerdo a su capacidad de almacenamiento, tiempos de respuesta, robustez, entre otros (Tuohy, 2013).

Codina (2010) sostiene que el motor de bases de datos preferente por los programadores web es MYSQL, gracias a que brinda facilidades de integración con otras arquitecturas y a la seguridad de la información. Sus principales fortalezas son: la rapidez, facilidad de adaptación a las aplicaciones web, no posee licenciamiento y puede ser utilizada en múltiples plataformas. Así, para el presente proyecto se utilizará el modelo entidad relación para la organización de las tablas del sistema. Montecino (2007) definen al modelo entidad-relación como un diagrama de ingeniería de software que se utiliza para desarrollar un modelo de datos de alta calidad y que paulatinamente se está convirtiendo en la técnica universal para el modelamiento de datos. También, este modelo ayuda a detectar sin ambigüedades los objetos que formarán parte de la base de datos, proporcionando una terminología adecuada para las fases de diseño y utiliza los conceptos de entidad, atributo y relación. Así, los autores González \& González (2013) sostienen que las características de un diagrama entidad relación deben ser claridad, coherencia, simplicidad y fidelidad en todos las entidades, atributos y relaciones que conforman la base de datos.

Un factor diferenciador en un sistema es la eficiencia, así lo sostiene Rueda (2011) cuando se refiere a ella como la 
forma de medir la utilización de los recursos asignados para cumplir un propósito $\mathrm{u}$ objetivo. Por ende, la determinación de la eficiencia en una solución tecnológica es importante para la toma de decisiones de un proceso. Para esto, Chirinos \& Padrón (2010) afirman que, para determinar la eficiencia de un sistema de inventario se debe medir los tiempos de ejecución del proceso de extracción, transformación y carga (ETL). Es decir, que este proceso es el encargado de canalizar los datos, depurarlos y finalmente cargarlos a la base de datos para su posterior utilización en la generación de reportes.

\section{Metodología}

El presente estudio posee un enfoque analítico descriptivo. Dado que, por medio de una fundación teórica sustenta el poder de contar con la tecnología RFID para la ejecución de una gestión de inventarios en la biblioteca de la IES.

Se realizará el desarrollo de un software que sea capaz de realizar la integración entre los equipos RFID y el sistema de administración de libros vigentes. La prueba tendrá como alcance las estanterías $1 \mathrm{~A}$ y $1 \mathrm{~B}$ con un total de 735 ejemplares, distribuido en 570 y 165 respectivamente; pertenecientes a la sección de las obras de referencia y actualmente cuentan con la etiqueta RFID adheridas a las cubiertas.El diseño de la investigación está segmentado en 3 fases: preparación de requerimientos, el desarrollo del sistema integrador y las pruebas de funcionamiento. A continuación un breve descripción de cada una de ellas.
Fase 1: Preparación de requerimientos.- En esta fase, se realizará el levantamiento de la información con las personas involucradas en el proceso de inventario de la biblioteca. Luego, se procederá a revisar la disponibilidad de los recursos técnicos necesarios para el desarrollo del sistema y se determinará la viabilidad del proyecto.

Fase 2: Desarrollo del sistema integrador.- Esta fase consiste en el desarrollo del sistema web que integrará la información determinada por dispositivos RFID y el software de administración bibliotecario de la IES.

Fase 3: Pruebas de Funcionamiento.En esta fase, se realizarán las pruebas de funcionamiento. Para ello, se realizará la instalación de los equipos RFID en la estación de trabajo de la biblioteca; así también se conectará el sistema web desarrollado con los equipos de RFID y finalmente también se instalará el sistema integrador en la estación en donde se encontrarán habilitados los equipos RFID. Con esto, se realizarán pruebas y evaluación de los módulos.

\section{Desarrollo}

\section{Preparación de requerimientos.}

A continuación, se exponen los requerimientos o funcionalidades que el sistema deberá poseer para resolver el problema de ejecución de inventario.

Lo primero a considerar es la generación de inventarios: esta funcionalidad permite generar de forma rápida una com- 
paración entre los datos capturados y los que han sido provistos del sistema de administración de la biblioteca de la IES.

Luego, se realiza la creación de perfiles de usuarios: esta funcionalidad permite crear y eliminar los perfiles de los usuarios que utilizarán en el sistema. Así también, permite otorgarle los permisos requeridos para cumplir sus funciones. Entonces, también se hace la generación de reportes estadísticos: esta funcionalidad permite realizar la visualización de forma básica en porcentaje los incrementos de los libros, en la base de datos del sistema de gestión bibliotecaria. Finalmente, se deberá realizar la generación de reportes históricos: esta funcionalidad permite visualizar reportes basándose en inventarios anteriores.

Para este proyecto, la IES provisionó de los equipos RFID, suministros y servidor para el desarrollo, pruebas e implementación, existiendo viabilidad en la ejecución del proyecto.

Las herramientas de programación son de desarrollo libre, por lo que no se requiere de licencia comercial para la instalación, son propias del programador.

A continuación, en la Figura 2 se muestra el Diagrama de Bloques del Proyecto mostrando la secuencia de actividades a realizar.

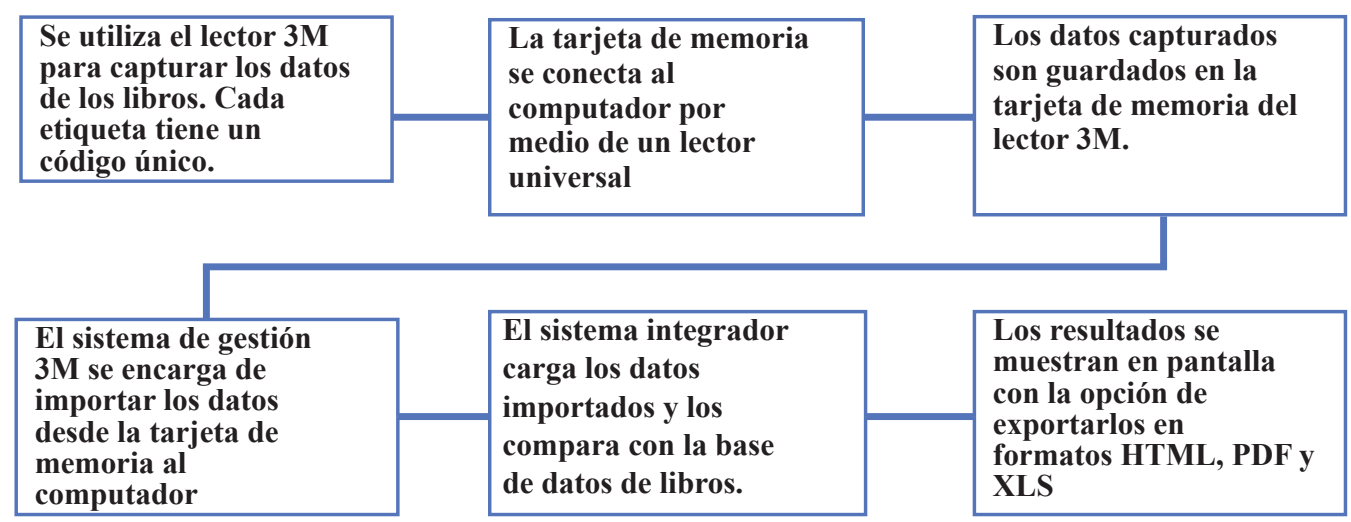

Figura 2. Diagrama de Bloques

Desarrollo de la aplicación.

En esta sección, se describe el diseño del software necesario para el sistema integrador de la biblioteca para el control de inventarios. Cabe recalcar que es un sistema que necesita la interacción con hardware, una interfaz del usuario y un servidor de datos. Estos dos componentes se instalarán y trabajarán en conjunto.
La interfaz al haber sido desarrollada en la web con el lenguaje PHP, puede ser ejecutada en cualquier navegador; siempre y cuando se encuentren dentro de la misma red. Para la elaboración del sistema integrador se seleccionó a Dreamweaver como herramienta de desarrollo, a PHP como lenguaje de programación y MYSQL como el motor de la base de datos. 
Conviene señalar que, la programación se realizará en módulos aplicando el modelo MVC para la reutilización del código y así agilitar los tiempos de procesamiento. Se instalará en un computador de escritorio el motor de base de datos, en donde los registros de la carga manual de libros y de los archivos capturados por el lector RFID se guardarán. A continuación, se muestran los parámetros de configuración más relevantes del sistema:

$\checkmark$ Nombre del Servidor: localhost

$\checkmark$ Puerto que trabaja: 3306

$\checkmark$ Nombre de la base de datos: biblioteca

$\checkmark$ Usuario: root

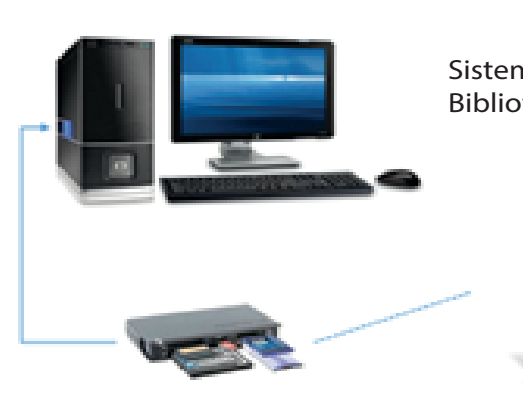

Lector de memoria universal

Proceso de transferencia de datos 2
Se debe de asegurar que, las configuraciones de MYSQL se encuentren configuradas por defecto. Por ende, solamente el administrador del sistema podrá tener acceso a la base de datos. El equipo en donde se instalará el sistema, la base de datos y sus componentes ejercerán como servidores y resto de computadores se podrán comunicar siempre y cuando se encuentren en la misma red y cuente con los permisos necesarios para ingresar a la aplicación.

En la Figura 3: Se muestra la representación gráfica del funcionamiento del sistema integrador de la biblioteca.
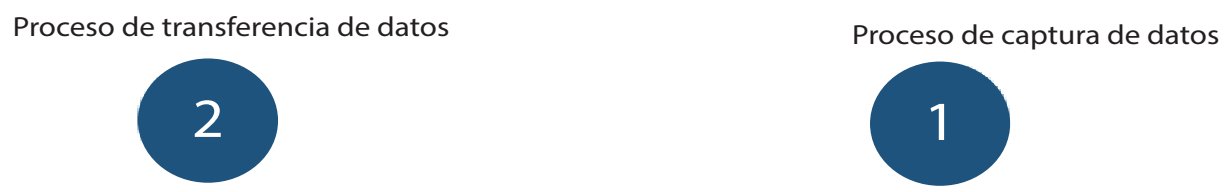

Figura 3. Funcionamiento del sistema con adaptación RFID

Para realizar el sistema, se utilizó el motor de base de datos MYSQL, el cual deberá de proporcionar un servicio confiable y estable. El manejador gráfico para la base de datos es PHP MyAdmin, el cual viene integrado en la solución WAMP (Windows, Apache, MYSQL, PHP). Las versiones instaladas en el servidor son: WAMP 2.1; Apache 2.2.17; PHP 5.3.4; MYSQL 5.1.53

La interfaz web es el complemento necesario para la utilización del sistema, para lo cual se tomaron en cuenta las exigencias planteadas en la fase de preparación de requerimientos, a fin que 
puedan satisfacer las necesidades planteadas. Con la interfaz de usuario, diseñada como página web, se garantiza que se pueda acceder desde cualquier equipo que se encuentre en la misma red.

Se debe de tener en cuenta que, en el equipo servidor debe encontrarse encendido e iniciados los servicios de Apache, MYSQL y PHP para que puedan funcionar los equipos en red. La interfaz web consta de varias secciones: pantalla de inicio, pantalla de menú, pantalla de configuraciones, pantalla de creación de usuarios, pantalla de inventario, pantalla de carga de base de datos, pantalla de historial de inventarios, pantalla de estadísticas, pantalla de reportes históricos y pantalla de reportes totales.

En la Figura 4: Se describe por medio del caso de uso las funcionalidades que tendrá el sistema integrador y los perfiles de cada uno de los operadores.

\section{Planificación de pruebas.}

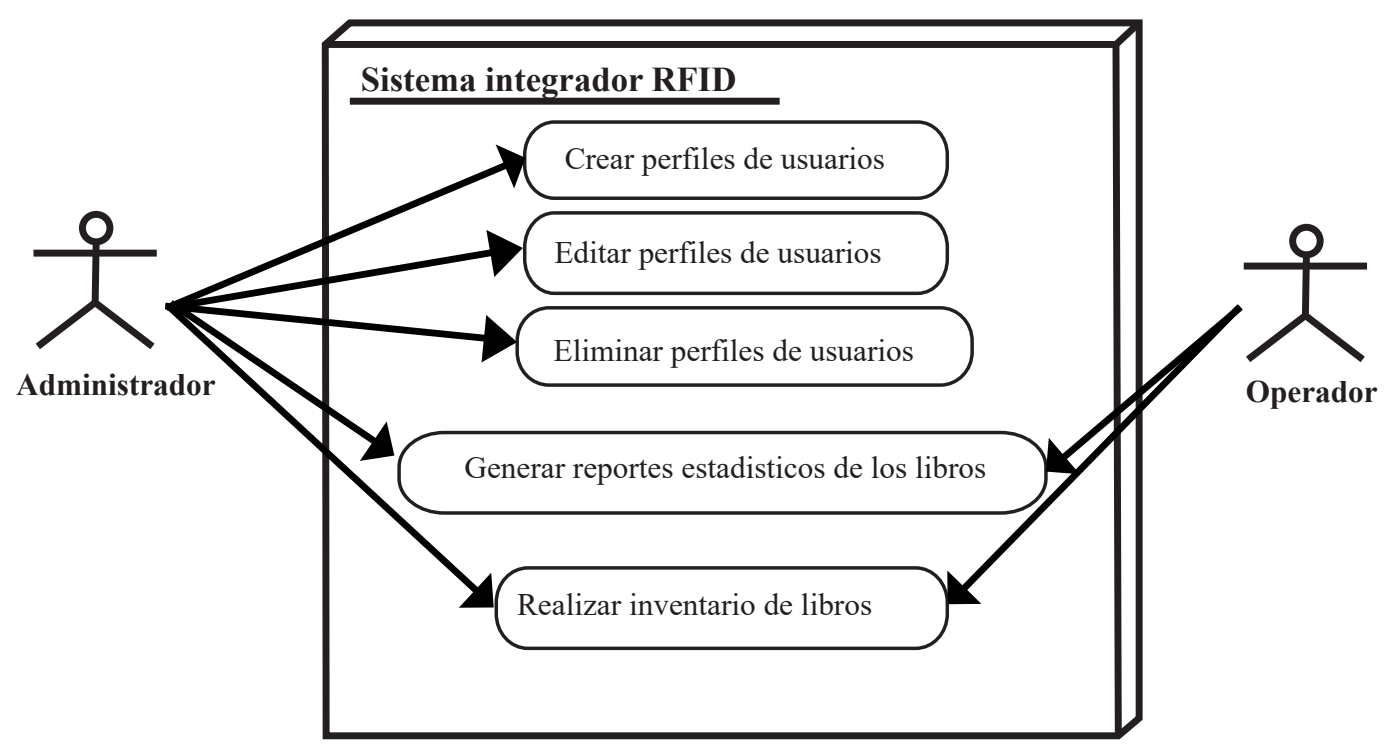

Figura 4. Caso de Uso Sistema Integrador

Como ya fue mencionado en la metodología, esta fase consiste en realizar las pruebas necesarias al sistema integrador, con la finalidad de validar el correcto funcionamiento del sistema y evitar cualquier incidencia en el ambiente de producción. Con el objetivo de realizar pruebas acertadas y lo más cercanas a la realidad, se contará con una base de datos alimentada con datos reales que posee la biblioteca de la Institución de Educación Superior.

Para el presente proyecto, el paráme- tro de evaluación principal es el tiempo de ejecución del proceso de inventario. Por lo tanto, el tiempo que habitualmente tarda en efectuar un inventario a una estantería se lo denomina "valor del parámetro inicial" y fue provisto mediante la consulta al personal encargado de realizar el proceso de inventario de la biblioteca.

Por otro lado, el parámetro de medición del mismo proceso utilizando el sistema y los equipos RFID se denomina "valor del parámetro final" y fue obtenido 
mediante la observación y la utilización de un cronómetro para medir la duración del proceso. Se obtuvieron resultados exitosos en la ejecución del proceso de generación de inventario, mejorando los tiempos de ejecución del proceso. Después de varias pruebas del proceso de inventario aleatorio (10 libros) con el sistema, se pudieron establecer valores que permitieron realizar una mejora en los tiempos de respuesta de la aplicación.

Inicialmente el tiempo de respuesta del sistema era de 11 minutos, lo que no era favorable, porque era semejante al tiempo que tomaba realizar el inventario de forma manual. Fue entonces, cuando se hizo una reutilización en el código, lo que permitió que se obtuviera una mejora sustancial en el tiempo final, obteniendo como resultado 4 minutos. Esto lo muestra en la figura 5.

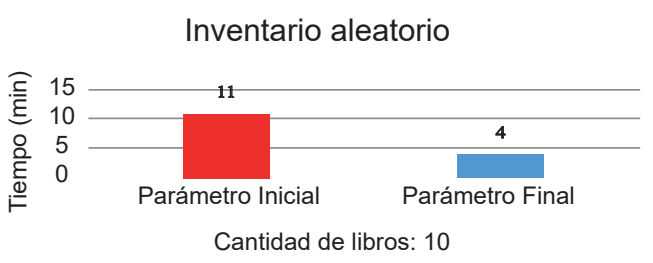

Figura 5. Prueba de inventario aleatorio. Implementación del Proyecto

Para realizar la implementación del sistema se deben de tener en cuenta los requerimientos mínimos de hardware y software, ya que el computador en donde se instalará el sistema también servirá de servidor para los demás equipos que estarán conectados en red.

\section{Requerimientos mínimos de hardware.}

a. PC con procesador Intel Core i5-3330 3.00 GHz.

b. Memoria RAM 3GB (3.90 GB utilizable)

c. Disco Duro con capacidad libre de $250 \mathrm{~Gb}$.

d. Instalación de lector RFID 3M modelo 803.

e. Instalación de lector universal de memoria.

\section{Requerimientos de software.}

a. Sistema operativo: Windows $7 \mathrm{de}$ 64bits.

b. Navegador web: Google Chrome, Internet Explorer o Firefox.

c. Instalación de aplicación $3 \mathrm{M}$ Conversion Station.

d. Instalación de aplicación 3M Digital Data Managert.

En la Figura 6 se muestran los equipos RFID instalados en la biblioteca.

\section{Pruebas y Resultados}

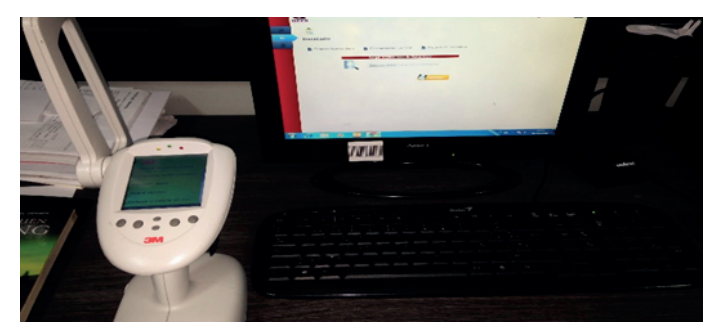

Figura 6. Instalación de equipos RFID

En la Figura 7, muestra la operatividad del sistema, mostrando la gráfica de las categorías del inventario.

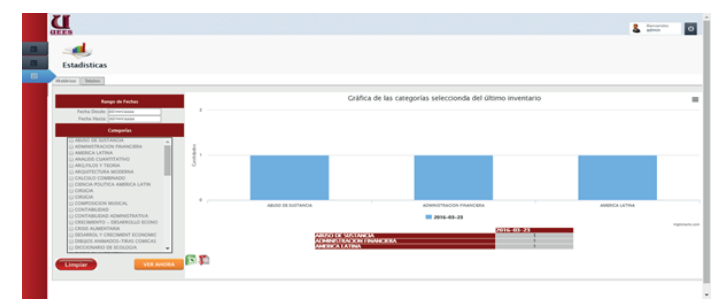

Figura 7. Operatividad del sistema, reportes estadísticos. 
Para comprobar la efectividad del sistema implementado, se realizaron pruebas enfocadas a administración de perfiles de usuario, así también al registro de inventario de ejemplares y al módulo de reportes estadísticos.

Prueba del Módulo de Administración para Creación de Perfiles

Como resultado de la prueba del módulo de administración de perfiles, se pudo realizar tanto la edición de los perfiles de usuarios, así como también la inactivación del mismo si se lo requiriera. Con estas pruebas se constató que el manejo del sistema era sencillo, que contaba con las seguridades respectivas teniendo validaciones de accesos y realizaba en forma correcta todos sus procesos.

Pruebas y Resultados del Módulo de Inventario

Esta prueba consiste en captura de datos de los libros y guardarlos en la memoria interna que utiliza el DLA del RFID, luego se coloca la memoria en el lector universal conectado a la computadora para la extracción.

Así también, se realizaron pruebas en el módulo del proceso de inventario donde se registraron los ejemplares de las estanterías. En las pruebas se pudo evidenciar que se cargó correctamente la base de datos de libros, así como también se cargó el archivo plano proveniente del DLA. Una vez realizadas las mencionadas cargas; se visualizó por pantalla todo el inventario registrado. Con esto, el módulo se podría presentar visualmente el historial de inventarios realizados, teniendo la opción a eliminarlos o imprimirlos.

Se demostró la eficiencia en la ejecución del proceso de inventario al medir el tiempo de ejecución del proceso manual con un cronómetro primeramente. Luego, se calculó el rendimiento del sistema integrador, para ello fue necesario realizar nuevamente el proceso de inventario, esta vez aplicando la tecnología RFID, obteniéndose tiempos de respuestas óptimos; tal como se muestra en la Tabla 1.

Por último, se realizaron pruebas al

Tabla 1.

Resultados de pruebas de sistema.

\begin{tabular}{|c|c|c|c|c|}
\hline Tareas del Inventario & $\begin{array}{l}\text { Cantidad } \\
\text { de } \\
\text { Libros }\end{array}$ & $\begin{array}{c}\text { Parámetro } \\
\text { Inicial }\end{array}$ & $\begin{array}{c}\text { Parámetro } \\
\text { Final }\end{array}$ & $\begin{array}{l}\text { Porcentaje } \\
\text { de mejora }\end{array}$ \\
\hline Inventario de una estantería $1^{a}$ & 570 & $190 \mathrm{~min}$ & $65 \min$ & $65,78 \%$ \\
\hline Inventario de una estantería 1B & 165 & $67 \mathrm{~min}$ & $18 \mathrm{~min}$ & $73,13 \%$ \\
\hline Inventario de libros (Prueba aleatorio) & 10 & $11 \mathrm{~min}$ & $4 \mathrm{~min}$ & $63,63 \%$ \\
\hline Elaboración de reportes de inventario & 10 & $25 \min$ & $3.5 \mathrm{~min}$ & $86 \%$ \\
\hline
\end{tabular}


proceso de generación de reportes estadísticos de los inventarios realizados, evidenciando que se podía imprimir de acuerdo a categorías seleccionadas y la alternativa de exportarlos en formato: xls, pdf o html.

\section{Conclusiones}

El desarrollo del proyecto evidenció que la tecnología RFID permitió realizar procesos de inventario, reduciendo procesos muertos, eliminación de errores de intervención humana y garantizando la fiabilidad de la información.

El utilizar el modelo MVC en el sistema integrador, estructurándolo por módulos, permitió la integración con las aplicaciones propietarias y los dispositivos RFID de una manera más eficiente.

Se constató con las pruebas realizadas, que la gestión de inventario para la biblioteca de IES mejoró en un promedio de $72 \%$, comprobando de esta manera lo expuesto en la literatura de este artículo, donde afirma, que tecnología de radiofrecuencia RFID permite optimizar recursos considerándola una solución rentable y adaptable para incrementar su productividad.

Así también existió limitación en cuanto al acceso del software de administración de biblioteca puesto que existían restricciones por seguridad de la información contenida en ella. Por tal motivo, la carga de la base de datos de libros se realizó de manera manual por medio de un archivo plano al mencionado sistema.

Para futuras investigaciones se podría estudiar la incidencia de software y tecnología de radiofrecuencia en la gestión de préstamos de libros. Además, de realizar una estadística de libros más prestados o más demandados con la implementación de aplicaciones web.

\section{Referencias}

Adhiarna, N., Hwang, Y.-M., \& Rho, J.-J. (2011). A Two Dimensional Framework for RFID Adoption and Diffusion: Strategic Implications for Developing Countries. Journal of Technology Management \& Innovation, 189-201.

Calabria, J. (2011). Construcción y poblamiento de un datawarehouse basado en el paradigma de bases de datos objeto relacional. Prospectiva, 69-77.

Camarena, J., Trueba, A., Martínez, M., \& López, M. d. (2012). Automatización de la codificación del patrón modelo vista controlador (MVC) en proyectos orientados a la Web. Ciencias Exactas y Aplicadas, 239-250.

Chirinos, N., \& Padrón, E. (2010). La eficiencia docente en la prática educativa. Revista de ciencias sociales, 481-492.

Codina, L. (2010). Metodología de análisis de sistemas de información y diseño de base de datos documentales: aspectos lógicos y funcionales. Anuari Socadi de Informació $i$ Documentació, 195-209. 
Correa, A., Álvarez, C., \& Gómez, R. (2010). Sistemas de identificación por radiofrecuencia, código de barras y su relación con la gestión de la cadena de suministro. Estudios Gerenciales, 115-141.

Cruz, L. (2012). El Vaticano controla mediante RFID a clero y empleados. Caracas: ID Noticias.

Davara, A. (2010). RFID. Tecnología del Futuro. RFIDPOINT. LA COMUNIDAD DE RFID EN LATINOAMÉRICA, 1-8.

Equipo Minero. (2014). Avances en Tecnología de Automatización Mejoran Utilización de Datos de Proceso en Tiempo Real. Equipo Minero, 38-39.

Fontalvo, T., Mendoza, A., \& Visbal, D. (2014). Eficiencia en los procesos logísticos en las empresas certificadas en basc medellín mediante análisis envolvente de datos. U.D.C.A Actualidad \& Divulgación Científica, 265-274.

García, J., Barbeau, M., \& Kranakis, E. (2010). Security threats on EPC based RFID systems. IEEE Computer Society, 1242-1244.

González, M., \& González, J. S. (2013). Aplicación del éstandar ISO/IEC 9126-3 en el modelo de datos concetual entidad-relación. Reista Facultad de Ingeniería UPTC, 113-125.
Guaman, C., \& Jorge Recalde. (2013). Implementación de un sistema informático para la automatización de los procesos de donaciones, registro de caja, control de pacientes e inventario, utilizando visual estudio express para el albergue FUDIS. Guayaquil: Puce.

Jabba, D., Alcocer, A., \& Rojas, C. (2011). Análisis comparativo de las herramientas de programación Web PHP, ASP y JSP, bajo los sistemas operativos Linux y Windows. Ingeniería y desarrollo revista de la División de Ingeniería de la Universidad del Norte, 104-115.

Jechlitschek, C. (2006). A Survey Paper on Radio Frecuency. MIchigan: Advanced Encryption Standar.

Jiménez, Y. (2013). RFID - EPC Código Electrónico de Producto como Herramienta de Control de Merma. INGE CUC, 11-20.

León, E. (2011). Estacionamiento automatizado con tecnología RFID. Cocniencia Tecnológica, 71-73.

Llamazares, J. C. (2013). Tarjetas identificadoras sin contacto o sistemas RFID. Mexico D.F: ecojovn.

Mallar, M. Á. (2010). PROCESS MANAGEMENT: AN EFFECTIVE MANAGEMENT 
APPROACH. Visión del Futuro, $1-19$.

Marimón, M. J. (2010). Innovación en la Bibliotecade ESADE: el proyecto de implementación de RFID. Dossier, 83-89.

Martínez, J. (2010). Fundamentos de programación de Java. G-TEC, $1-206$.

Mejía, S., Yepes, D., \& Arango, B. (2013). Gestión e Implementación del RFID en las Empresas. Gestión de las Personas y Tecnología, 49-56.

Meliá, J. (2011). Lightweight PRG for Low-Cost Passive RFID Security Improvement. Universitat Orbeta de Catalunya. Internet Interdisciplinary Institute (IN3), 26-36.

Montecino, A. (2007). Proyecto de integración de sistemas de archivos Linux - MySQL. Científica, 7-11.

Muñuzuri, J., Escudero, A., Rodríguez, M., \& Rubia, C. (2012). Evaluación y respuesta a los problemas y fallos de implantación en un proyecto piloto industrial de identificación por radio frecuencia (RFID). DYNA, 593-600.

Ramírez, E., \& Meléndez, F. (2014). Sistemas RFID aplicados al control de grandes inventarios. Inge@Juan-Tendencias en la Ingeniería, 69-80.
Rubinos, A., \& Nuevo, H. (2011).

Seguridad en bases de datos. Revista Cubana de Ciencias Informáticas, 1-16.

Rueda, N. (2011). La eficiencia y su importancia en el sector público. eXtoikos, 38-47.

Sierra, R. (2012). Implantación de un sistema RFID para obtener trazabilidad en la cadena de suministros. Cataluña: Upcommons.

Sistema de Gestion. (2015). Ventajas de la tecnología RFID versus el Código de Barras. Cataluña: Ingenieria Electrónica.

Sistemas Informaticos. (2012). Captura automatizada de datos . Madrid: Informatica Avanzada.

Swedberg, C. (2016). Meggitt Polymers \& Composites Used RFID to Track Aircraft Components. Sweedem: RFID Journal.

Texas Instrument. (2014). Near Field Communication. USA: Texas Instrument.

Texas Instrument. (2015). Stellaris RFID Wilreless Kit. USA: Texas Instrument.

Tuohy, P. (2013). The RPG vs. PHP Debate. Ipro Developer, 14-17.

Villareal, E., Arango, D., \& Moreno, D. (2012). Prototipo para el control y ubicación de artículos en inventa- 
rios por medio de un carro grúa y RFID. Prospectiva (Una nueva visión para la ingeniería), 18-27.

Villarejo, N. (2010). Propuesta de un archivo virtual dinámico en PHP para gestionar la documentación electoral manuscrita del Archivo Histórico del Congreso de los Diputados de España. Documentación de las ciencias de la información, 329-348.

Wei, G., \& Rui, H. (2013). Design and Implementation of Competitiveness Evaluation System for Metallurgical Enterprises Based on MySQL and JSP. Journal of Convergence Information Technology, $1-10$.

Zapata, C., González, G., \& Chaverra, J. J. (2011). Generación automática del diagrama entidad-relación y su representación en sql desde un lenguaje controlado (un-lencep). Revista Ingenierías Universidad de Medellín, 127-136. 
